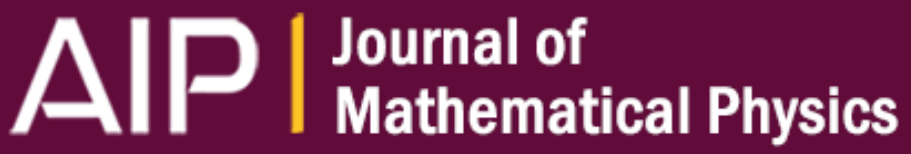

\section{Critical behavior in Angelesco ensembles}

K. Deschout and A. B. J. Kuijlaars

Citation: Journal of Mathematical Physics 53, 123523 (2012); doi: 10.1063/1.4769822

View online: http://dx.doi.org/10.1063/1.4769822

View Table of Contents: http://scitation.aip.org/content/aip/journal/jmp/53/12?ver=pdfcov

Published by the AIP Publishing

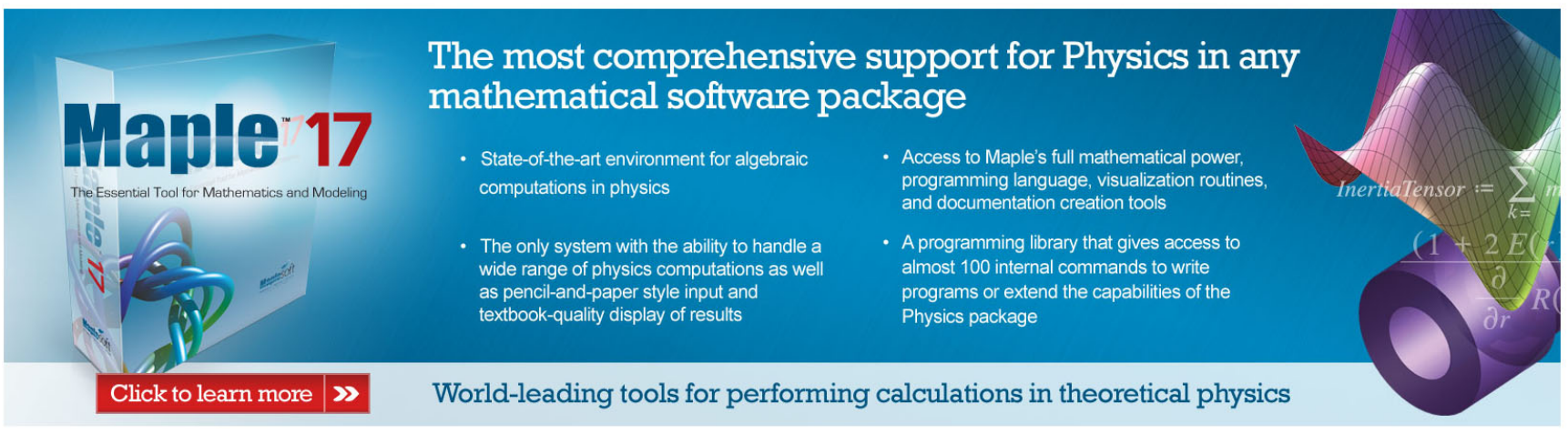




\title{
Critical behavior in Angelesco ensembles
}

\author{
K. Deschout ${ }^{\mathrm{a})}$ and A. B. J. Kuijlaars ${ }^{\text {b) }}$ \\ Department of Mathematics, Katholieke Universiteit Leuven, Celestijnenlaan 200B, \\ 3001 Leuven, Belgium
}

(Received 12 March 2012; accepted 5 November 2012; published online 14 December 2012)

\begin{abstract}
We consider Angelesco ensembles with respect to two modified Jacobi weights on touching intervals $[a, 0]$ and $[0,1]$, for $a<0$. As $a \rightarrow-1$ the particles around 0 experience a phase transition. This transition is studied in a double scaling limit, where we let the number of particles of the ensemble tend to infinity while the parameter $a$ tends to -1 at a rate of $\mathcal{O}\left(n^{-1 / 2}\right)$. The correlation kernel converges, in this regime, to a new kind of universal kernel, the Angelesco kernel $\mathbb{K}^{\text {Ang. The }}$ result follows from the Deift/Zhou steepest descent analysis, applied to the RiemannHilbert problem for multiple orthogonal polynomials. (ㅇ 2012 American Institute of Physics. [http://dx.doi.org/10.1063/1.4769822]
\end{abstract}

\section{INTRODUCTION AND STATEMENT OF RESULTS}

Multiple orthogonal polynomial (MOP) ensembles ${ }^{21}$ form an extension of the more familiar orthogonal polynomial (OP) ensembles..$^{20}$ The latter appears as the eigenvalue distributions of unitary random matrix ensembles.

A major topic of interest in random matrix theory deals with the universality conjecture. This conjecture claims that, as the size of the matrices tends to infinity, the local eigenvalue statistics of a random matrix ensemble converge to universal limits, i.e., limits that are independent of the precise probability distribution on the matrices. The limits only depend on macroscopic eigenvalue characteristics and on the symmetry class of the matrix ensemble.

For the class of unitary random matrix ensembles the problem concerning universality can be translated into a problem concerning the associated sequence of OP. By the Gaudin-Mehta method $^{16,17,25}$ the eigenvalue density function can be written as a determinantal point process. The relevant correlation kernel is the OP kernel, which is also known as the Christoffel-Darboux kernel. Universality is then expressed as the convergence of this OP kernel to a certain universal limiting kernel. This convergence can be derived by considering the large degree asymptotics of the OP. Common limiting kernels include the sine, Airy, and Bessel kernels.

For other random matrix ensembles a connection exists with MOP: the eigenvalues are a determinantal point process with the so-called MOP kernel as correlation kernel. A prime example of such a connection occurs in the unitary random matrix model with external source. By analysis of the MOP kernel in this model universality has been shown and the sine and Airy kernels appeared., ${ }^{2,5}$ However, in a critical case this model also exhibits behavior that does not appear in OP ensembles: under certain scaling the MOP kernel converges to the so-called Pearcey kernel. ${ }^{6,8,29}$ MOP ensembles also gave rise to new critical behavior in the papers. ${ }^{14,23}$

This is a recurring feature: MOP ensembles can exhibit a wider variety of critical phenomena than OP ensembles. In this paper we consider another type of MOP ensembles, the so-called Angelesco ensembles. These ensembles do not appear in a natural way as the eigenvalue process of a random matrix ensemble, but the notion of universality still makes sense. We show that a new kind of critical behavior occurs in Angelesco systems, determined by a new kind of universal kernel, that we call the Angelesco kernel $\mathbb{K}^{\text {Ang }}$.

\footnotetext{
a)klaas.deschout@gmail.com.

b)arno.kuijlaars@wis.kuleuven.be.
} 
An Angelesco system of weights ${ }^{1,26}$ is a system of $r \geq 2$ weights $\vec{w}=\left(w_{1}, \ldots, w_{r}\right)$ on the real line such that the supports of the weights are contained in intervals with pairwise disjoint interiors. Thus there exist bounded real intervals $\Delta_{1}, \ldots, \Delta_{r}$ such that

$$
\stackrel{\circ}{\Delta}_{j} \cap \stackrel{\circ}{\Delta}_{k}=\varnothing \quad \text { for } j \neq k
$$

and $w_{j}$ is non-negative and integrable on the real line with

$$
w_{j}(x) \equiv 0 \quad \text { for } x \in \mathbb{R} \backslash \Delta_{j} .
$$

Angelesco ensembles are MOP ensembles associated with Angelesco systems of weights. Let $\vec{n}$ be a multi-index $\left(n_{1}, \ldots, n_{r}\right) \in \mathbb{N}^{r}$ and define $|\vec{n}|:=n_{1}+\cdots+n_{r}$. The Angelesco ensemble with respect to $\vec{w}$ and $\vec{n}$ is the probability measure on $\mathbb{R}^{|\vec{n}|}$ with probability density function

$$
\mathcal{P}_{\vec{n}}\left(x_{1}, \ldots, x_{|\vec{n}|}\right):=\frac{1}{Z_{\vec{n}}} \operatorname{det}\left[x_{k}^{j-1}\right]_{j, k=1}^{|\vec{n}|} \cdot \operatorname{det}\left[f_{j}\left(x_{k}\right)\right]_{j, k=1}^{|\vec{n}|},
$$

where $Z_{\vec{n}}$ is a normalization constant and

$$
f_{n_{1}+\cdots+n_{i-1}+j}(x):=x^{j-1} w_{i}(x) \text { for } j=1, \ldots, n_{i}, i=1, \ldots, r .
$$

The Angelesco properties (1.1) and (1.2) imply that (1.3) is non-negative for every $\left(x_{1}, \ldots, x_{|\vec{n}|}\right)$ $\in \mathbb{R}^{|\vec{n}|}$, and can be non-zero only if $n_{j}$ of the particles $x_{1}, \ldots, x_{|\vec{n}|}$ are in $\Delta_{j}$ for every $j=1, \ldots, r$. Thus in an Angelesco ensemble, with probability $1, n_{j}$ particles are located in $\Delta_{j}$ for every $j=1$, $\ldots, r$.

The probability density function (1.3) is a biorthogonal ensemble, ${ }^{7}$ which is a special case of a determinantal point process. The correlation kernel is

$$
K_{\vec{n}}(x, y)=\sum_{j=1}^{|\vec{n}|} \sum_{k=1}^{|\vec{n}|}\left(M^{-1}\right)_{k, j} x^{j-1} f_{k}(y),
$$

where $\left(M^{-1}\right)_{k, j}$ is the $k j$ th entry of the inverse of the matrix

$$
M=\left(m_{j, k}\right)_{j, k=1}^{|\vec{n}|}, \quad m_{j, k}=\int x^{j-1} f_{k}(x) d x .
$$

That is, see Refs. 4 and 21 ,

$$
\mathcal{P}_{\vec{n}}\left(x_{1}, \ldots, x_{|\vec{n}|}\right)=\frac{1}{|\vec{n}| !} \operatorname{det}\left[K_{\vec{n}}\left(x_{j}, x_{k}\right)\right]_{j, k=1}^{|\vec{n}|},
$$

and for each $m=1, \ldots,|\vec{n}|$,

$$
\rho_{m}\left(x_{1}, \ldots, x_{m}\right)=\operatorname{det}\left[K_{\vec{n}}\left(x_{j}, x_{k}\right)\right]_{j, k=1}^{m},
$$

where $\rho_{m}$ is the $m$-point correlation function. Another representation for $K_{\vec{n}}$ is by means of a determinant

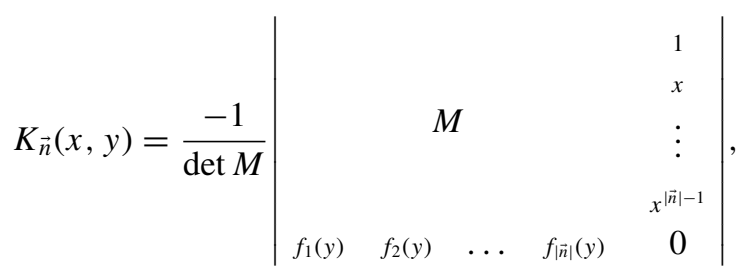

where $M$ is the moment matrix (1.6).

In this paper we consider Angelesco ensembles with respect to two weights $w_{1}$ and $w_{2}$. Also we restrict ourselves to diagonal multi-indices $\vec{n}=(n, n)$. Our interest is in the local asymptotics of the MOP kernel $K_{\vec{n}}=K_{n, n}$ associated to the weights $w_{1}$ and $w_{2}$. By rescaling if necessary we can assume that $\Delta_{1}=[a, b]$ and $\Delta_{2}=[0,1]$ for some $a<b \leq 0$.

The type of local behavior around a certain point is suggested by the behavior of the limiting mean particle density in that point. Assume that $w_{j}>0$ almost every on $\Delta_{j}$. Then this limiting density only depends on the endpoints of the two intervals $\Delta_{1}$ and $\Delta_{2}$. Depending on the values of 


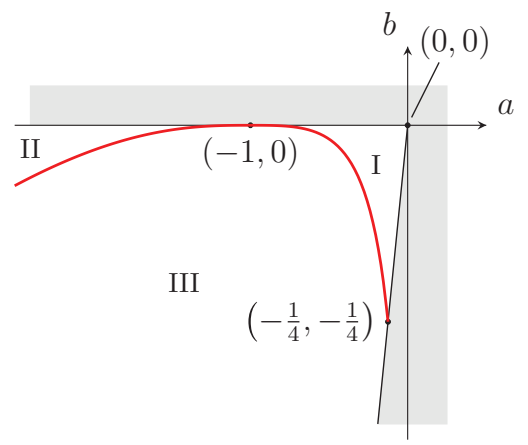

FIG. 1. The phase diagram for Angelesco ensembles with two weights, supported on $[a, b]$ and $[0,1]$. The gray areas represent values of $a$ and $b$ that are forbidden because of the restriction $a<b \leq 0$. Remark that the scale on the two axes is different.

$a$ and $b$ we get a qualitatively different picture. We can distinguish 3 cases and a number of phase transitions, see Figure 1.

Case III corresponds to relatively large separation between the two intervals. As a consequence there are hard edges in all four endpoints $a, b, 0$, and 1 , meaning that the limiting particle distribution blows up like an inverse square root. After rescaling around one of these endpoints the MOP kernels converge to limiting kernels that depend on the behavior of the weight at that endpoint. In the typical case of Jacobi-type weight functions

$$
\begin{aligned}
& w_{1}(x)=(x-a)^{\alpha}(b-x)^{\beta} h_{1}(x) \chi_{[a, b]}(x), \\
& w_{2}(x)=x^{\gamma}(1-x)^{\delta} h_{2}(x) \chi_{[0,1]}(x),
\end{aligned}
$$

this leads to Bessel kernels.

In the cases I and II the separation is small. A gap will emerge on the larger of the two intervals $\Delta_{1}$ and $\Delta_{2}$, due to the pushing of the zeros effect. There will then be three hard edges, and one soft edge, where the limiting particle density vanishes like a square root. Around the soft edge the rescaled MOP kernel will converge to the Airy kernel (in the case of weights (1.9)).

The transition between case III and cases I and II is probably related to the Painlevé II equation as in Ref. 9. The Bessel, Airy, and Painlevé II kernels already appear as scaling limits for OP ensembles. ${ }^{15}$

This paper deals with the critical point $(a, b)=(-1,0)$, where the two intervals $\Delta_{1}$ and $\Delta_{2}$ are touching and of equal size. In this case the limiting particle density behaves like $|x|^{-\frac{1}{3}}$ as $x \rightarrow 0$, see Refs. 13 and 19. We study this critical case in a double scaling limit, where we put $b=0$ and let $a$ tend to -1 as $n \rightarrow \infty$,

$$
a=a_{n}:=-1+\frac{\sqrt{2} \tau}{n^{\frac{1}{2}}} \quad \text { for } \tau \in \mathbb{R} .
$$

The parameter $\tau$ is known as the double scaling parameter. This scaling regime corresponds to the phase transition between cases I and II with $b$ fixed at 0 .

For $a \neq-1$ and $b=0$ we have a gap in the spectrum between 0 and $s$ where $s=s_{a}$ is given by

$$
s_{a}=\frac{(a+1)^{3}}{9\left(a^{2}-a+1\right)},
$$

see Ref. 19. In $s$ a soft edge appears, while in 0 we have a hard edge. Plots of the limiting particle densities in the cases $a<-1, a=-1$, and $a>-1$ are given in Figure 2. As $a$ tends to -1 the gap closes and the soft edge meets with the hard edge. This soft-to-hard-edge collision will give rise to new critical behavior in 0 .

So for the remainder of the paper we take for $a<0$

$$
\Delta_{1}:=[a, 0], \quad \Delta_{2}:=[0,1],
$$




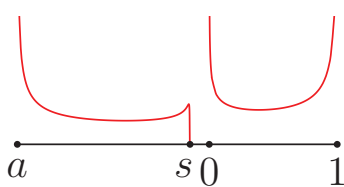

$$
\begin{gathered}
a<-1 \\
(a=-1.5)
\end{gathered}
$$

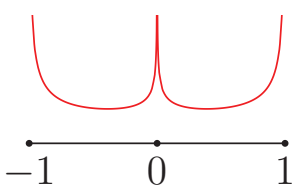

$a=-1$

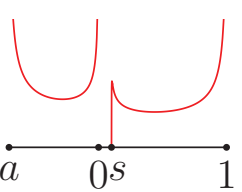

$$
a>-1
$$$$
(a=-0.7)
$$

FIG. 2. Plot of the limiting particle densities in Angelesco ensembles with two weights on $[a, 0]$ and $[0,1]$ in the cases $a<-1, a=-1$, and $-1<a<0$. For clarity the size of the gap between 0 and $s$ has been exaggerated in the figure. In the left and right plots the density has a local maximum near $s$. Even though the plots may suggest otherwise, the density is in fact real analytic there.

and as in Ref. 13 we consider the following modified Jacobi weights:

$$
\begin{aligned}
& w_{1}(x):=(x-a)^{\alpha}|x|^{\beta} h_{1}(x) \chi_{[a, 0]}(x), \\
& w_{2}(x):=x^{\beta}(1-x)^{\gamma} h_{2}(x) \chi_{[0,1]}(x),
\end{aligned}
$$

with $\alpha, \beta, \gamma>-1$ and functions $h_{j}$ that are positive on $\Delta_{j}$ and analytic in a neighborhood of $\Delta$ for $j=1,2$. Note that we use the same exponent $\beta$ in both $w_{1}$ and $w_{2}$.

In Ref. 13 we considered the weights (1.13) and obtained a Mehler-Heine asymptotic formula for the MOP associated to $w_{1}$ and $w_{2}$ and $\vec{n}=(n, n)$ as $n \rightarrow \infty$. See also Refs. 27 and 28 .

We use the notation $K_{n, n}(\cdot, \cdot ; a)$ to denote the dependence of the correlation kernel (1.5) on the parameter $a$. The main result is then as follows:

Theorem 1.1: Let $K_{n, n}$ be the correlation kernel of the MOP ensemble with weights (1.13) and diagonal multi-index $\vec{n}=(n, n)$. Let $\tau, x, y \in \mathbb{R}$ with $y \neq 0$. Then as $n \rightarrow+\infty$ we have

$$
\frac{1}{\sqrt{2} n^{\frac{3}{2}}} K_{n, n}\left(\frac{x}{\sqrt{2} n^{\frac{3}{2}}}, \frac{y}{\sqrt{2} n^{\frac{3}{2}}} ;-1+\frac{\sqrt{2} \tau}{n^{\frac{1}{2}}}\right)=\mathbb{K}^{\mathrm{Ang}}(x, y ; \tau)+\mathcal{O}\left(\frac{y^{\beta}}{n^{\frac{1}{6}}}\right),
$$

for some limiting kernel $\mathbb{K}^{\mathrm{Ang}}(x, y ; \tau)$ that will be described below in Propositions 1.2 and 1.3. The $\mathcal{O}$-term in (1.14) is uniform for $x$ in a bounded set and $y$ in a compact subset in $\mathbb{R} \backslash\{0\}$.

The limiting kernel $\mathbb{K}^{\text {Ang }}$ is referred to as the Angelesco kernel. It has an expression in terms of the Angelesco model parametrix $\Psi$ which we will describe in Sec. II B. More explicit expressions exist, one involving a certain pairing of two analytic functions $q_{0}, r_{0}$ and one in terms of a double contour integral.

Proposition 1.2: Define functions $q_{0}$ and $r_{0}$ by

$$
\begin{array}{ll}
q_{0}(x)=q_{0}(x ; \tau):=\frac{1}{2 \pi i}|x|^{\beta+2} \int_{\Gamma_{0}} t^{-\beta-3} e^{\frac{\tau x}{t}-\frac{x^{2}}{2 t^{2}}+t} d t, & x \in \mathbb{R}, \\
r_{0}(y)=r_{0}(y ; \tau):=\frac{1}{2 \pi i}|y|^{-\beta-1} \int_{\widehat{\Gamma}_{0}} s^{\beta} e^{-\frac{\tau y}{s}+\frac{y^{2}}{2 s^{2}}-s} d s, & y \in \mathbb{R} \backslash\{0\} .
\end{array}
$$

Here the contours $\Gamma_{0}$ and $\widehat{\Gamma}_{0}$ are shown in Figure 3, and we choose the principal branches for the fractional powers $t \mapsto t^{-\beta-3}$ and $s \mapsto s^{\beta}$. Then we can write $\mathbb{K}^{\mathrm{Ang}}(x, y ; \tau)$ as a pairing of $q_{0}(x)$ and $r_{0}(y)$ in the following way:

$$
\mathbb{K}^{\mathrm{Ang}}(x, y ; \tau)=\left|\frac{y}{x}\right|^{\beta}\left[\begin{array}{c}
y r_{0}(y) q_{0}^{\prime \prime}(x)-\left((\beta+1) r_{0}(y)+y r_{0}^{\prime}(y)\right) q_{0}^{\prime}(x) \\
+\left(y r_{0}^{\prime \prime}(y)+(\beta+2) r_{0}^{\prime}(y)-\tau r_{0}(y)\right) q_{0}(x)
\end{array}\right] .
$$

From the expressions for $q_{0}$ and $r_{0}$ it is clear that we have

$$
q_{0}(x ; \tau)=q_{0}(-x ;-\tau), \quad r_{0}(y ; \tau)=r_{0}(-y ;-\tau) .
$$




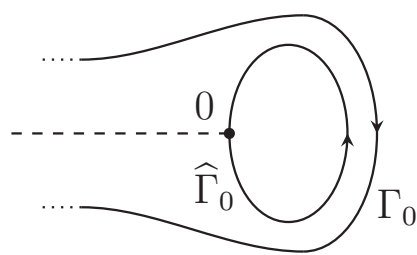

FIG. 3. The contours $\Gamma_{0}$ and $\widehat{\Gamma}_{0}$ appearing in the integral formulas (1.15), (1.16), and (1.20) for the Angelesco kernel $\mathbb{K}^{\text {Ang }}$. The dashed line denotes the branch cut of $t^{\beta}$ and $s^{\beta}$.

Hence the Angelesco kernel satisfies the following (expected) symmetry:

$$
\mathbb{K}^{\mathrm{Ang}}(x, y ; \tau)=\mathbb{K}^{\mathrm{Ang}}(-x,-y ;-\tau) .
$$

Proposition 1.3: Let the contours $\Gamma_{0}$ and $\widehat{\Gamma}_{0}$ be again as in Figure 3, and take $x, y \in \mathbb{R} \backslash\{0\}$, $\tau \in \mathbb{R}$. Then we have the following double integral formula for $\mathbb{K}^{\text {Ang }}(x, y ; \tau)$ :

$$
\mathbb{K}^{\text {Ang }}(x, y ; \tau)=\frac{\operatorname{sgn}(y)}{(2 \pi i)^{2}} \int_{t \in \Gamma_{0}} \int_{s \in \widehat{\Gamma}_{0}} \frac{s^{\beta}}{t^{\beta}} \frac{1}{x s-y t} \frac{e^{\frac{\tau x}{t}-\frac{x^{2}}{2 t^{2}}+t}}{e^{\frac{\tau y}{s}-\frac{y^{2}}{2 s^{2}}+s}} d s d t .
$$

Again main branches are used for the fractional powers $t \mapsto t^{\beta}$ and $s \mapsto s^{\beta}$.

Remark 1.4: In Refs. 22 and 23 the authors consider a model of $n$ non-intersecting squared Bessel paths, conditioned to start at time $t=0$ in the point $x=a>0$, and to end at time $t=1$ at $x=0$, see Figure 4. For each fixed time $t$ the particles form a MOP ensemble with respect to orthogonality weights involving the modified Bessel functions $I_{\beta}$ and $I_{\beta+1}$ for some $\beta>-1$.

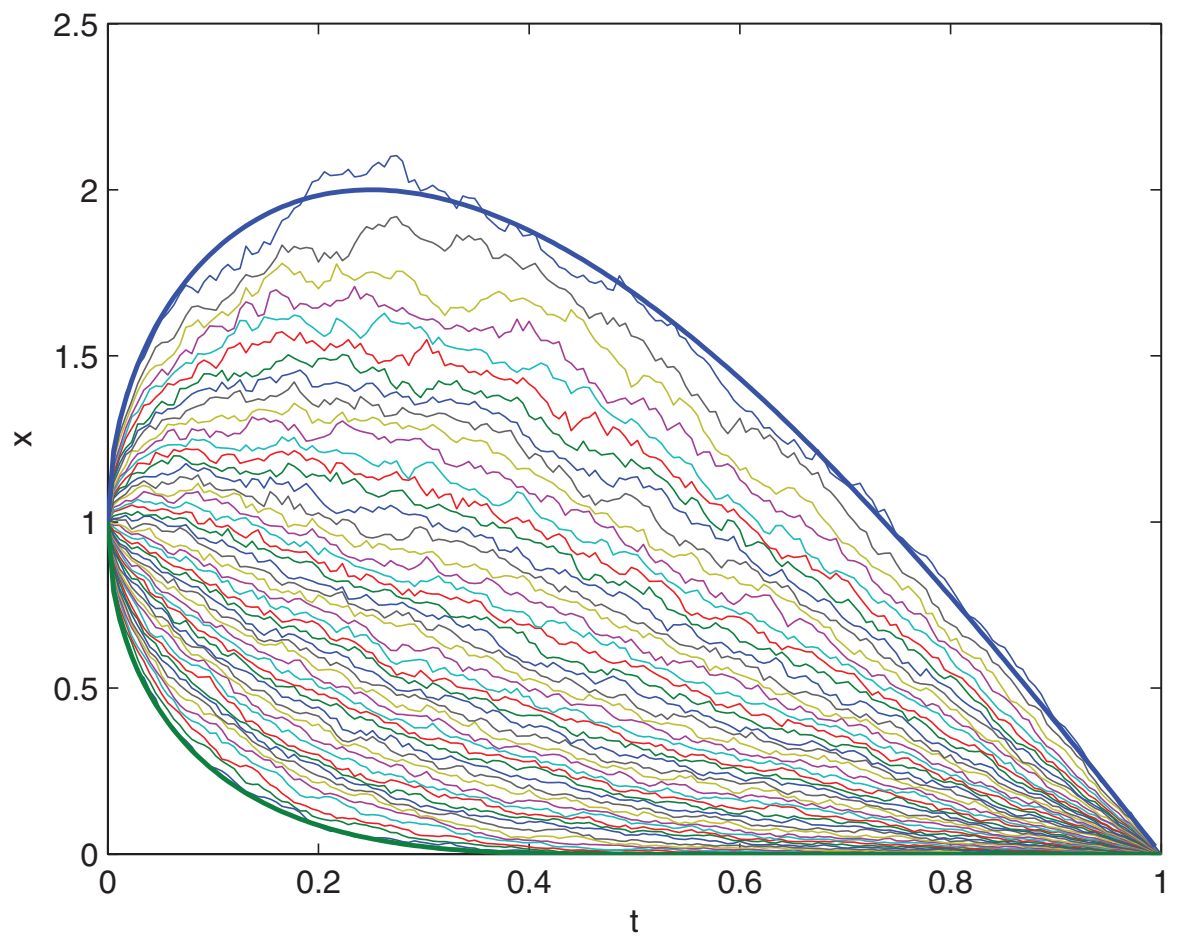

FIG. 4. Numerical simulation of 50 non-intersecting squared Bessel paths. On the horizontal axis, time is running from 0 to 1 . The vertical axis has the position $x$. The figure is taken from Ref. 22 . 
Associated to each fixed time $t$ one has a limiting mean particle distribution as $n \rightarrow \infty$. For small values of $t$ the particles are supported on an interval in $\mathbb{R}^{+}$, away from 0 . On the other hand, for values of $t$ near 1 the point 0 is contained in the support, meaning that particles stick to the hard wall in 0 . There is then a critical time $t=t^{*} \in(0,1)$ at which the gap between the particles and the wall in 0 closes. This phase transition is similar to the phase transition in Angelesco ensembles.

By taking a double scaling limit around $x=0$ and $t=t^{*}$ a new kind of universal limiting kernel, $K^{\text {crit }}$, was obtained (Eq. (1.18) of Ref. 23). The function $K^{\text {crit }}$ depends on two positive position variables $x$ and $y$, a double scaling parameter $\tau$, and the parameter $\beta$ appearing in the modified Bessel functions. It turns out that this critical kernel is almost the same as the Angelesco kernel $\mathbb{K}^{\text {Ang }}$. By applying a simple substitution to the double integral formula for $K^{\text {crit }}$ (Eq. (1.19) of Ref. 23) one obtains the following identity:

$$
\mathbb{K}_{\beta}^{\text {Ang }}(x, y ; \tau)=\frac{y^{\beta}}{x^{\beta}} K_{\beta}^{c r i t}(y, x ; \tau), \quad x, y>0,
$$

where we used the notation $\mathbb{K}_{\beta}^{\text {Ang }}$ to indicate the dependence of the kernel on the parameter $\beta$.

Although the kernels are different this identity implies an equality at the level of the correlation functions. Hence, after proper identification of the parameters, the critical behavior in Angelesco ensembles coincides with the critical behavior in the model of non-intersecting squared Bessel paths. A significant difference between the two models is that in the squared Bessel paths model the particles remain on the positive half-line, while in our model they are located on both sides of 0 and there is interaction between the two groups.

The proof of Theorem 1.1 follows from the application of the Deift/Zhou steepest descent method on the Riemann-Hilbert (RH) problem for MOPs. ${ }^{11,12}$ In Sec. II we recall the RH formulation for the weights $w_{1}$ and $w_{2}$ (1.13) and we give a formula for the MOP kernel in terms of the RH problem for MOPs. Also we recall the RH problem for the Angelesco local model parametrix $\Psi$ which is taken from Ref. 13.

The Deift/Zhou method consists of a sequence of invertible transformations reducing the original RH problem into a normalized RH problem, for which uniform estimates can be made. The analysis is the same as in Ref. 13. Instead of giving full details we will, in Sec. III, give a quick overview of the transformations and describe their effect on the expression for the MOP kernel.

In Sec. IV we shall use the series of transformations to show that the limit in Theorem 1.1 indeed holds, and we give expressions for the Angelesco kernel $\mathbb{K}^{\text {Ang }}$ in terms of the Angelesco parametrix $\Psi$.

Finally, Sec. V contains the proofs of the two explicit expressions for $\mathbb{K}^{\text {Ang }}$ stated in Propositions 1.2 and 1.3.

\section{RIEMANN-HILBERT PROBLEM}

This section recalls the RH characterization of the relevant multiple orthogonal polynomials and the Angelesco local model parametrix.

\section{A. Multiple orthogonal polynomials}

We state here the RH problem for MOPs ${ }^{30}$ with respect to the system of weights $\left(w_{1}, w_{2}\right)$ given in (1.13) and the multi-index $\left(n_{1}, n_{2}\right) \in \mathbb{N}^{2}$. The endpoint conditions are as in Ref. 24.

We look for a $3 \times 3$ matrix-valued function $Y$ such that:

- $Y$ is defined and analytic on $\mathbb{C} \backslash[a, 1]$.

- $Y$ has continuous boundary values $Y_{ \pm}$on $(a, 0)$ and $(0,1)$ and they satisfy the jump relation $Y_{+}=Y_{-} J_{Y}$ with $J_{Y}$ given by

$$
J_{Y}(x)=\left(\begin{array}{ccc}
1 & w_{1}(x) & w_{2}(x) \\
0 & 1 & 0 \\
0 & 0 & 1
\end{array}\right), \quad x \in(a, 0) \cup(0,1) .
$$


Recall that $w_{1}(x) \equiv 0$ outside of $[a, 0]$ and $w_{2}(x) \equiv 0$ outside of $[0,1]$.

- As $z \rightarrow \infty$ we have

$$
Y(z)=\left(I+\mathcal{O}\left(\frac{1}{z}\right)\right)\left(\begin{array}{ccc}
z^{n_{1}+n_{2}} & 0 & 0 \\
0 & z^{-n_{1}} & 0 \\
0 & 0 & z^{-n_{2}}
\end{array}\right) .
$$

- $Y$ has the following behavior at the endpoints of the intervals:

$$
\begin{aligned}
& Y(z)=\mathcal{O}\left(\begin{array}{lll}
1 & \epsilon(z) & 1 \\
1 & \epsilon(z) & 1 \\
1 & \epsilon(z) & 1
\end{array}\right), \text { as } z \rightarrow a, \\
& \text { where } \epsilon(z)= \begin{cases}(z-a)^{\alpha} & \text { if } \alpha<0, \\
\log (z-a) & \text { if } \alpha=0, \\
1 & \text { if } \alpha>0\end{cases} \\
& Y(z)=\mathcal{O}\left(\begin{array}{ccc}
1 & 1 & \epsilon(z) \\
1 & 1 & \epsilon(z) \\
1 & 1 & \epsilon(z)
\end{array}\right) \text {, as } z \rightarrow 1 \text {, } \\
& \text { where } \epsilon(z)= \begin{cases}(z-1)^{\gamma} & \text { if } \gamma<0, \\
\log (z-1) & \text { if } \gamma=0, \\
1 & \text { if } \gamma>0 .\end{cases} \\
& Y(z)=\mathcal{O}\left(\begin{array}{ccc}
1 & \epsilon(z) & \epsilon(z) \\
1 & \epsilon(z) & \epsilon(z) \\
1 & \epsilon(z) & \epsilon(z)
\end{array}\right), \text { as } z \rightarrow 0 \\
& \text { where } \epsilon(z)= \begin{cases}z^{\beta} & \text { if } \beta<0 \\
\log z & \text { if } \beta=0, \\
1 & \text { if } \beta>0\end{cases}
\end{aligned}
$$

The $\mathcal{O}$-symbol is to be taken entry-wise.

This RH problem has a unique solution in terms of MOP with respect to the weights $w_{1}$ and $w_{2}$. In particular we have that $Y_{11}(z)$ is a monic polynomial of degree $n_{1}+n_{2}$ that satisfies the multiple orthogonality conditions

$$
\int x^{k} Y_{11}(x) w_{j}(x) d x=0, \quad k=0, \ldots, n_{j}-1, \quad j=1,2 .
$$

For the full expression of $Y$ we refer to Ref. 13. Most important for the present paper is the following formula for the MOP correlation kernel in terms of $Y$, see Refs. 4 and 10:

$$
K_{n_{1}, n_{2}}(x, y)=\frac{1}{2 \pi i(x-y)}\left(\begin{array}{lll}
0 & w_{1}(y) & \left.w_{2}(y)\right) Y_{+}(y)^{-1} Y_{+}(x)
\end{array}\left(\begin{array}{l}
1 \\
0 \\
0
\end{array}\right) .\right.
$$

This formula is valid for $x \neq y \in \mathbb{R}$ with $y \notin\{a, 0,1\}$. As before it is understood that $w_{1}(y) \equiv 0$ for $y$ outside $(a, 0)$, and $w_{2}(y) \equiv 0$ for $y$ outside $(0,1)$. The formula $(2.6)$ can be extended to the case $x=y \notin\{a, 0,1\}$ by l'Hôpital's rule. 


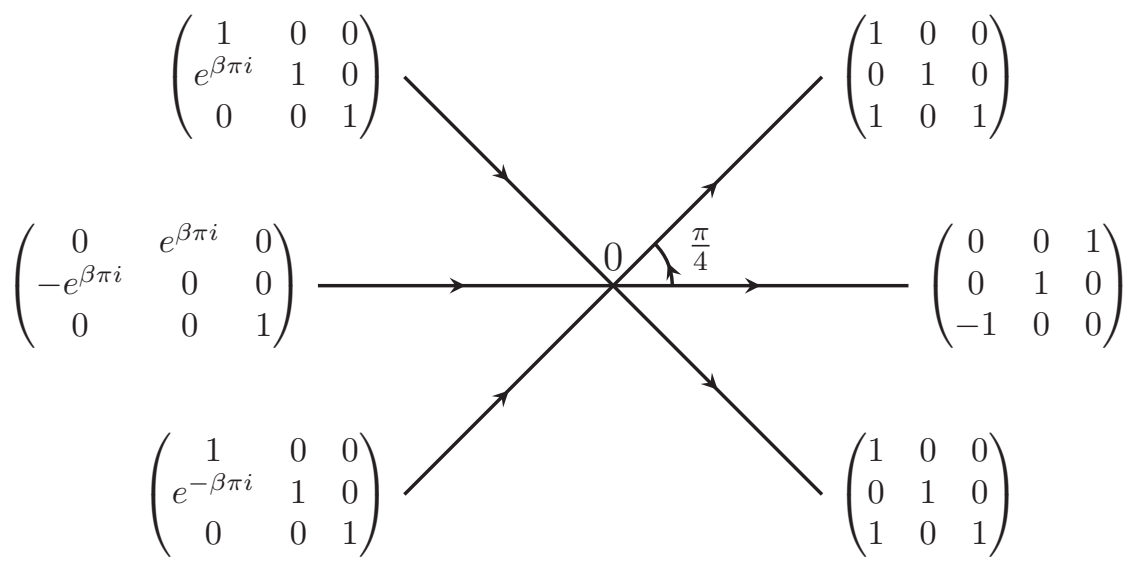

FIG. 5. The contour $\Sigma_{\Psi}$ and the jump matrices of $\Psi$.

\section{B. Angelesco local model parametrix}

At a crucial step in the steepest descent analysis we need to do a local analysis at the point 0 . This step involves certain special functions, which are combined into a $3 \times 3$ matrix-valued function, called the Angelesco model parametrix $\Psi$ and which was introduced in Sec. 2.2 of Ref. 13. The function $\Psi$ depends on two parameters $\beta>-1$ and $\tau \in \mathbb{R}$. Since $\beta$ is considered fixed we do not emphasize the dependence on $\beta$. We may write $\Psi(z ; \tau)$ to emphasize the dependence on $\tau$.

The function $\Psi$ has the following RH characterization:

- $\Psi$ is defined and analytic on $\mathbb{C} \backslash \Sigma_{\Psi}$ where $\Sigma_{\Psi}$ is a contour consisting of six oriented rays through the origin, as shown in Figure 5.

- $\Psi$ has continuous boundary values on $\Sigma_{\Psi} \backslash\{0\}$ that satisfy the jump condition

$$
\Psi_{+}(z)=\Psi_{-}(z) J_{\Psi}(z) \quad z \in \Sigma_{\Psi} \backslash\{0\},
$$

where the jump matrices $J_{\Psi}$ are also given in Figure 5 .

- Denote $\omega:=e^{2 \pi i / 3}$. As $z \rightarrow \infty$ with $\pm \operatorname{Im} z>0$, we have

$$
\Psi(z)=\sqrt{\frac{2 \pi}{3}} e^{\frac{\tau^{2}}{6}} z^{\frac{\beta}{3}}\left(\begin{array}{ccc}
z^{\frac{1}{3}} & 0 & 0 \\
0 & 1 & 0 \\
0 & 0 & z^{-\frac{1}{3}}
\end{array}\right) L_{ \pm}\left(I+\mathcal{O}\left(z^{-\frac{1}{3}}\right)\right) B_{ \pm} e^{\Theta(z ; \tau)},
$$

where $L_{ \pm}, B_{ \pm}$, and $\Theta(z ; \tau)$ are defined by

$$
\begin{aligned}
& L_{+}:=\left(\begin{array}{ccc}
-\omega^{2} & 1 & \omega \\
1 & -1 & -1 \\
-\omega & 1 & \omega^{2}
\end{array}\right), \quad B_{+}:=\left(\begin{array}{ccc}
e^{\frac{\beta \pi i}{3}} & 0 & 0 \\
0 & 1 & 0 \\
0 & 0 & e^{-\frac{\beta \pi i}{3}}
\end{array}\right), \\
& L_{-}:=\left(\begin{array}{ccc}
\omega & 1 & \omega^{2} \\
-1 & -1 & -1 \\
\omega^{2} & 1 & \omega
\end{array}\right), \quad B_{-}:=\left(\begin{array}{ccc}
e^{-\frac{\beta \pi i}{3}} & 0 & 0 \\
0 & 1 & 0 \\
0 & 0 & e^{\frac{\beta \pi i}{3}}
\end{array}\right), \\
& \Theta(z ; \tau):= \begin{cases}\operatorname{diag}\left(\theta_{1}(z ; \tau), \theta_{3}(z ; \tau), \theta_{2}(z ; \tau)\right) & \text { for } \operatorname{Im} z>0, \\
\operatorname{diag}\left(\theta_{2}(z ; \tau), \theta_{3}(z ; \tau), \theta_{1}(z ; \tau)\right) & \text { for } \operatorname{Im} z<0,\end{cases}
\end{aligned}
$$

and the $\theta_{k}$ are defined by

$$
\theta_{k}(z ; \tau):=-\frac{3}{2} \omega^{k} z^{\frac{2}{3}}-\tau \omega^{2 k} z^{\frac{1}{3}} \quad \text { for } k=1,2,3 .
$$


The expansion (2.7) for $\Psi(z)$ as $z \rightarrow \infty$ is valid uniformly for $\tau$ in a bounded set.

- Denote by $s_{1}, s_{2}$ the sectors

$$
\begin{aligned}
& s_{1}:=\left\{z \in \mathbb{C} \mid \arg z \in\left(-\frac{3 \pi}{4},-\frac{\pi}{4}\right) \cup\left(\frac{\pi}{4}, \frac{3 \pi}{4}\right)\right\}, \\
& s_{2}:=\left\{z \in \mathbb{C} \mid \arg z \in\left(-\frac{\pi}{4}, \frac{\pi}{4}\right) \cup\left(\frac{3 \pi}{4}, \frac{5 \pi}{4}\right)\right\} .
\end{aligned}
$$

Around 0 we then have the following estimate:

$$
\begin{aligned}
\Psi(z)=\mathcal{O}\left(\begin{array}{lll}
\epsilon_{1}(z) & \epsilon_{2}(z) & \epsilon_{2}(z) \\
\epsilon_{1}(z) & \epsilon_{2}(z) & \epsilon_{2}(z) \\
\epsilon_{1}(z) & \epsilon_{2}(z) & \epsilon_{2}(z)
\end{array}\right) \text { as } z \rightarrow 0, \\
\text { with }\left(\epsilon_{1}(z), \epsilon_{2}(z)\right)= \begin{cases}\left(z^{\beta}, z^{\beta}\right) & \text { for } \beta<0, \\
(1, \log z) & \text { for } \beta=0, z \in s_{1}, \\
(\log z, \log z) & \text { for } \beta=0, z \in s_{2}, \\
\left(z^{\beta}, 1\right) & \text { for } \beta>0, z \in s_{1}, \\
(1,1) & \text { for } \beta>0, z \in s_{2} .\end{cases}
\end{aligned}
$$

Note that the parameter $\beta$ appears in the jump condition and in the behavior around 0 . The dependence on $\tau$ is only in the asymptotic condition as $z \rightarrow \infty$.

The RH problem for $\Psi$ has a unique solution. It is constructed out of solutions of the third order linear differential equation

$$
z q^{\prime \prime \prime}(z)-\beta q^{\prime \prime}(z)-\tau q^{\prime}(z)+q(z)=0 .
$$

This differential equation (2.13) has solutions in the form of contour integrals

$$
q(z)=\int_{\Gamma} t^{-\beta-3} e^{\frac{\tau}{t}-\frac{1}{2 t^{2}}+z t} d t
$$

where $\Gamma$ is an appropriate contour so that the integrand vanishes at the endpoints of the contour $\Gamma$. Define three contours $\Gamma_{1}, \Gamma_{2}$, and $\Gamma_{3}$ as in Figure 6, and define for $z$ with $\operatorname{Re} z>0$

$$
q_{j}(z):=\int_{\Gamma_{j}} t^{-\beta-3} e^{\frac{\tau}{t}-\frac{1}{2 t^{2}}+z t} d t, \quad j=1,2,3,
$$

where we choose the branch of $t^{-\beta-3}$ with a cut on the positive real axis, i.e.,

$$
t^{-\beta-3}=|t|^{-\beta-3} e^{(-\beta-3) i \arg t}, \quad 0<\arg t<2 \pi .
$$

The integrals (2.15) only converge for $z$ with $\operatorname{Re} z>0$, but the functions $q_{j}$ can be continued analytically using contour deformations. Branch points for the $q$-functions are 0 and $\infty$ and we take the analytic continuation to $\mathbb{C} \backslash(-\infty, 0]$, thus with a branch cut on the negative real axis.

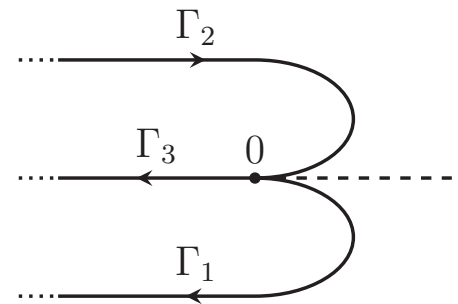

FIG. 6. The contours $\Gamma_{1}, \Gamma_{2}$, and $\Gamma_{3}$ in the $t$-plane. The dashed line denotes the cut of $t^{-\beta-3}$. 
In the upper half-plane the unique solution for $\Psi$ is then given by, see Ref. 13 for more details,

$$
\Psi:=\left\{\begin{array}{lll}
\left(\begin{array}{lll}
e^{2 \beta \pi i} q_{1} & e^{\beta \pi i} q_{3} & q_{2} \\
e^{2 \beta \pi i} q_{1}^{\prime} & e^{\beta \pi i} q_{3}^{\prime} & q_{2}^{\prime} \\
e^{2 \beta \pi i} q_{1}^{\prime \prime} & e^{\beta \pi i} q_{3}^{\prime \prime} & q_{2}^{\prime \prime}
\end{array}\right), & 0<\arg z<\frac{\pi}{4}, \\
\left(\begin{array}{llll}
e^{2 \beta \pi i} q_{1}+q_{2} & e^{\beta \pi i} q_{3} & q_{2} \\
e^{2 \beta \pi i} q_{1}^{\prime}+q_{2}^{\prime} & e^{\beta \pi i} q_{3}^{\prime} & q_{2}^{\prime} \\
e^{2 \beta \pi i} q_{1}^{\prime \prime}+q_{2}^{\prime \prime} & e^{\beta \pi i} q_{3}^{\prime \prime} & q_{2}^{\prime \prime}
\end{array}\right), & \frac{\pi}{4}<\arg z<\frac{3 \pi}{4}, \\
\left(\begin{array}{cccc}
e^{2 \beta \pi i} q_{1}+q_{2}-e^{2 \beta \pi i} q_{3} & e^{\beta \pi i} q_{3} & q_{2} \\
e^{2 \beta \pi i} q_{1}^{\prime}+q_{2}^{\prime}-e^{2 \beta \pi i} q_{3}^{\prime} & e^{\beta \pi i} q_{3}^{\prime} & q_{2}^{\prime} \\
e^{2 \beta \pi i} q_{1}^{\prime \prime}+q_{2}^{\prime \prime}-e^{2 \beta \pi i} q_{3}^{\prime \prime} & e^{\beta \pi i} q_{3}^{\prime \prime} & q_{2}^{\prime \prime}
\end{array}\right), & \frac{3 \pi}{4}<\arg z<\pi,
\end{array}\right.
$$

and in the lower half-plane by

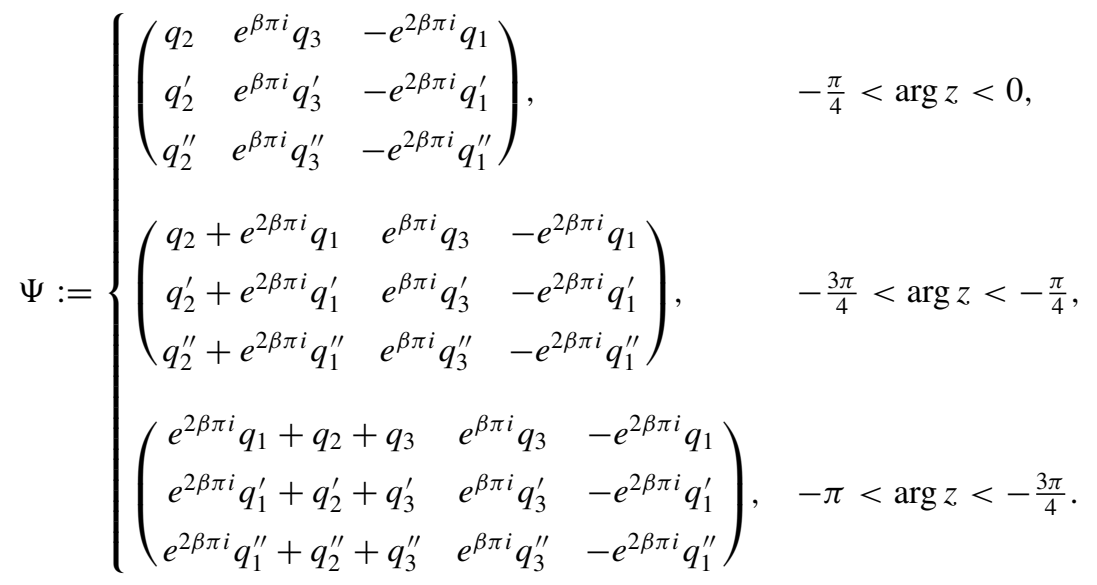

\section{STEEPEST DESCENT TRANSFORMATIONS}

In this section we apply the Deift/Zhou steepest descent method to the RH problem for $Y$. For details of the various transformations we refer to Ref. 13.

The Deift/Zhou method consists of a number of invertible transformations reducing the original matrix-valued function $Y$ to a function $R$ that is uniformly close to the identity matrix. Of main importance is the effect of these transformations on the expression for the MOP kernel $K_{n_{1}, n_{2}}(x, y)$, see (2.6), with diagonal multi-indices,

$$
n_{1}=n=n_{2} .
$$

The first transformation $Y \mapsto T$ normalizes the RH problem for $Y$ at infinity: the new matrixvalued function $T$ tends to the identity matrix at infinity. The transformation uses functions $g_{1}$ and $g_{2}$ derived from a modified equilibrium problem with logarithmic potentials. These $g$-functions are analytic on $\mathbb{C} \backslash(-\infty, 1]$. See Sec. 4.1 of Ref. 13 for precise details on the transformation $Y \mapsto T$. For the MOP kernel we obtain from (2.6) and formula (4.4) in Ref. 13

$$
\begin{aligned}
K_{n, n}(x, y)=\frac{1}{2 \pi i(x-y)}\left(\begin{array}{c}
0 \\
w_{1}(y) e^{2 n\left(g_{1,+}(y)+l_{1}+\pi i / 2\right)} \\
w_{2}(y) e^{2 n\left(g_{2,+}(y)+l_{2}\right)}
\end{array}\right)^{\mathrm{T}} & \\
& \times T_{+}(y)^{-1} T_{+}(x)\left(\begin{array}{c}
e^{2 n\left(g_{1,+}(x)+g_{2,+}(x)\right)} \\
0 \\
0
\end{array}\right) .
\end{aligned}
$$


The constants $l_{1}$ and $l_{2}$ appear in the Euler-Lagrange variational conditions related to the equilibrium problem.

The second transformation $T \mapsto S$ is the opening of the lenses, see Sec. 4.2 of Ref. 13. In this transformation the rapidly oscillating jump matrices along $(a, 0)$ and $(0,1)$ that appeared after the transformation $Y \mapsto T$ are turned into constant jump matrices, with the side effect of creating exponentially small jump matrices on the lips of the lenses. The expression for this transformation depends on the location of $z$. From now on we restrict ourselves to positive $x$ and $y$, since in the cases $x<0$ or $y<0$ the expressions for $K_{n, n}(x, y)$ are different but similar. Remark that we have $w_{1}(y)=0$ for $y>0$. By (3.2) and formulas (4.14) and (4.5) in Ref. 13, we then get for $x, y \in(0,1)$, (note that $w_{1}(y)=0$ ),

$$
\begin{aligned}
K_{n, n}(x, y)=\frac{1}{2 \pi i(x-y)}\left(\begin{array}{c}
-e^{-2 n\left(g_{1,+}(y)+g_{2,+}(y)\right)} \\
0 \\
w_{2}(y) e^{2 n\left(g_{2,+}(y)+l_{2}\right)}
\end{array}\right)^{\mathrm{T}} \\
\quad \times S_{+}(y)^{-1} S_{+}(x)\left(\begin{array}{c}
e^{2 n\left(g_{1,+}(x)+g_{2,+}(x)\right)} \\
0 \\
w_{2}(x)^{-1} e^{-2 n\left(g_{2,+}(x)+l_{2}\right)}
\end{array}\right) .
\end{aligned}
$$

The next two transformations $S \mapsto R_{0}$ and $R_{0} \mapsto R$ are applied after constructing local and global parametrices. These parametrices are built as approximations to the $\mathrm{RH}$ problem for $S$, and one can think of $R_{0}$ as the approximation error. The approximation around 0 is not good enough for the further analysis, whence the need for a very last transformation $R_{0} \mapsto R$. We refer to Sec. 4.4 of Ref. 13 for precise details and formulas.

The local parametrix $P_{0}$ around 0 is constructed on a disk $U_{0}$ centered in zero with shrinking radius $n^{-1 / 2}$, see formulas (4.68) and (4.70) of Ref. 13. It involves the Angelesco model parametrix $\Psi$ described in Sec. II B. Assume that $x$ and $y$ are positive and belong to the disk $U_{0}$. Then $S=R_{0} P_{0}$ and inserting this in (3.3) we obtain after some calculations

$$
\begin{aligned}
K_{n, n}(x, y)=\frac{1}{2 \pi i(x-y)} & \left|\frac{y^{\beta}}{x^{\beta}}\right|\left(\begin{array}{lll}
-1 & 0 & 1
\end{array}\right) \Psi_{+}\left(n^{\frac{3}{2}} f(y) ; n^{\frac{1}{2}} \tau(y)\right)^{-1} \\
& \times E_{n}(y)^{-1} R_{0}(y)^{-1} R_{0}(x) E_{n}(x) \Psi_{+}\left(n^{\frac{3}{2}} f(x) ; n^{\frac{1}{2}} \tau(x)\right)\left(\begin{array}{l}
1 \\
0 \\
1
\end{array}\right) .
\end{aligned}
$$

\begin{tabular}{|c|c|c|c|c|c|c|}
\hline & \multicolumn{3}{|c|}{$x<0$} & \multicolumn{3}{|c|}{$x>0$} \\
\hline$y<0$ & $(-1$ & $10)$, & $\left(\begin{array}{l}1 \\
1 \\
0\end{array}\right)$ & $(-1$ & $10)$, & $\left(\begin{array}{l}1 \\
0 \\
1\end{array}\right)$ \\
\hline & $(-1$ & $\left.\begin{array}{ll}0 & 1\end{array}\right)$, & 1 & $(-1$ & $\left.\begin{array}{ll}0 & 1\end{array}\right)$, & $\left(\begin{array}{l}0 \\
1\end{array}\right)$ \\
\hline
\end{tabular}

For $x<0$ or $y<0$ the formula (3.4) also holds, but with different row and column vectors, as given in the following table:

The functions $f$ and $\tau$ appearing in (3.4) were defined in Ref. 13. Since we do not need to know their full expressions we will restrict ourselves to recalling their most important properties.

These functions are both analytic in a neighborhood of 0 , with $f(0)=0$. They depend on $a$, and as $a \rightarrow-1$ one has

$$
f^{\prime}(0 ; a)=\sqrt{2}+\mathcal{O}(a+1), \quad \tau(0 ; a)=\frac{1}{\sqrt{2}}(a+1)+\mathcal{O}(a+1)^{2},
$$

see formula (4.76) of Ref. 13. From this it easily follows that 
Lemma 3.1: Let $x \in \mathbb{R} \backslash\{0\}$ and $\tau \in \mathbb{R}$. Put

$$
x_{n}:=\frac{x}{\sqrt{2} n^{\frac{3}{2}}}, \quad a_{n}:=-1+\frac{\sqrt{2} \tau}{n^{\frac{1}{2}}} .
$$

Then as $n \rightarrow \infty$ we have

$$
\begin{aligned}
& n^{\frac{3}{2}} f\left(x_{n} ; a_{n}\right)=x+\mathcal{O}\left(n^{-\frac{1}{2}}\right), \\
& n^{\frac{1}{2}} \tau\left(x_{n} ; a_{n}\right)=\tau+\mathcal{O}\left(n^{-\frac{1}{2}}\right) .
\end{aligned}
$$

The $\mathcal{O}$-term is uniform for $x$ in a bounded set.

Proof: This follows easily from (3.6) and the fact that $f(z ; a)$ and $\tau(z, a)$ converge uniformly as $a \rightarrow-1$ to $f(z ;-1)$ and $\tau(z ;-1)$.

Also we have the following technical estimate:

Proposition 3.2: Let $x, y \in \mathbb{R} \backslash\{0\}, \tau \in \mathbb{R}$ and put

$$
x_{n}:=\frac{x}{\sqrt{2} n^{\frac{3}{2}}}, \quad y_{n}:=\frac{y}{\sqrt{2} n^{\frac{3}{2}}}, \quad a_{n}:=-1+\frac{\sqrt{2} \tau}{n^{\frac{1}{2}}} .
$$

Then as $n \rightarrow \infty$ we have

$$
E_{n}\left(y_{n} ; a_{n}\right)^{-1} R_{0}\left(y_{n}\right)^{-1} R_{0}\left(x_{n}\right) E_{n}\left(x_{n} ; a_{n}\right)=I+\mathcal{O}\left(n^{-\frac{1}{6}}\right) .
$$

The $\mathcal{O}$-term is uniform for $x$ and $y$ in bounded sets.

Assuming this proposition we can finish the proof of Theorem 1.1 as follows. Let $x, y>0$ and let $x_{n}, y_{n}, a_{n}$ be as in (3.10). Then for $n$ large enough we have that $x_{n}, y_{n} \in U_{0}$. Using (3.8), (3.9), and (3.11) in (3.4) we then obtain

$$
\begin{aligned}
& \frac{1}{\sqrt{2} n^{\frac{3}{2}}} K_{n, n}\left(x_{n}, y_{n} ; a_{n}\right)= \\
& \frac{1}{2 \pi i(x-y)}\left|\frac{y}{x}\right|^{\beta}\left(\begin{array}{ccc}
-1 & 0 & 1
\end{array}\right) \Psi(y ; \tau)^{-1} \Psi(x ; \tau)\left(\begin{array}{l}
1 \\
0 \\
1
\end{array}\right)+\mathcal{O}\left(\frac{y^{\beta}}{n^{\frac{1}{6}}}\right) .
\end{aligned}
$$

This proves Theorem 1.1 for $x, y>0$. The right-hand side of (3.12) also provides us with an expression for $\mathbb{K}^{\mathrm{Ang}}(x, y ; \tau)$ in terms of the Angelesco parametrix $\Psi$. For $x<0$ or $y<0$ the proof is completely similar and (3.12) holds, but with different row and column vectors, as given in (3.5).

\section{PROOF OF PROPOSITION 3.2}

Proof: The proof of Proposition 3.2 is rather technical and requires deeper knowledge of the structure of the matrix functions $R_{0}$ and $E_{n}$.

From Sec. 5.2 of Ref. 13 we have

$$
R_{0}(z)=R(z)\left(I-V_{n}(z ; a)\right), \quad z \in U_{0},
$$

where, see formula (5.13) of Ref. 13,

$$
R(z)=I+\mathcal{O}\left(n^{-1 / 6}\right) \quad \text { as } n \rightarrow \infty,
$$

uniformly in $z$. The function $V_{n}$ in (4.1) is given by

$$
V_{n}(z ; a)=Z_{n}(z ; a)-\frac{Z_{n}^{(0)}(a)}{z},
$$


where $Z_{n}$ and $Z_{n}^{(0)}(a)$ play a role in the analysis of Ref. 13. The function $Z_{n}$ is analytic on $U_{0} \backslash\{0\}$ with a simple pole in 0 , whose residue is $Z_{n}^{(0)}(a)$. Hence $V_{n}$ is analytic on $U_{0}$. Additionally, from Eq. (4.84) of Ref. 13 it follows that $V_{n}$ is of the form

$$
V_{n}=\left(\begin{array}{c}
\text { scalar analytic } \\
\text { function in } z
\end{array}\right) \times D_{\infty}^{-1}\left(\begin{array}{c}
\sqrt{2} i \\
1 \\
-1
\end{array}\right)\left(\begin{array}{lll}
\sqrt{2} i & 1 & -1
\end{array}\right) D_{\infty}
$$

for a certain constant diagonal matrix $D_{\infty}$. As a consequence we have the following basic property of $V_{n}$ :

$$
V_{n}(x ; a) V_{n}(y ; a)=0, \quad \text { for every } x, y \in U_{0} .
$$

Additionally, $V_{n}\left(z ; a_{n}\right)$ is uniformly bounded on the disk $U_{0}$. Since $U_{0}$ has radius $n^{-1 / 2}$ this easily implies that $V_{n}^{\prime}\left(z_{n} ; a_{n}\right)=\mathcal{O}\left(n^{1 / 2}\right)$ if $z_{n}=\mathcal{O}\left(n^{-3 / 2}\right)$, and therefore

$$
V_{n}\left(x_{n} ; a_{n}\right)-V_{n}\left(y_{n} ; a_{n}\right)=\mathcal{O}\left(n^{-1}\right) \text {. }
$$

From (4.1) and the fact that $x_{n}=\mathcal{O}\left(n^{-3 / 2}\right), y_{n}=\mathcal{O}\left(n^{-3 / 2}\right)$, it follows that

$$
R\left(y_{n}\right)^{-1} R\left(x_{n}\right)=I+\mathcal{O}\left(n^{-\frac{7}{6}}\right) .
$$

Combining (4.7), (4.2), (4.5), and (4.6) we obtain

$$
R_{0}\left(y_{n}\right)^{-1} R_{0}\left(x_{n}\right)=I+\mathcal{O}\left(n^{-1}\right) .
$$

The matrix-valued function $E_{n}$ defined in formula (4.80) of Ref. 13 is analytic and invertible on a fixed neighborhood of 0 . It depends on both $a$ and $n$. It takes the form

$$
E_{n}(z ; a)=n^{-\frac{\beta}{2}} e^{-\frac{n \tau(z ; a)^{2}}{6}} \widetilde{E}(z ; a)\left(\begin{array}{ccc}
n^{-\frac{1}{2}} & 0 & 0 \\
0 & 1 & 0 \\
0 & 0 & n^{\frac{1}{2}}
\end{array}\right),
$$

where $\widetilde{E}$ is independent of $n$, and $\widetilde{E}$ converges uniformly as $a \rightarrow-1$. Then

$$
\widetilde{E}\left(y_{n} ; a_{n}\right)^{-1} \widetilde{E}\left(x_{n} ; a_{n}\right)=I+\mathcal{O}\left(n^{-\frac{3}{2}}\right),
$$

and combining with (4.9) yields

$$
E_{n}\left(y_{n} ; a_{n}\right)^{-1} E_{n}\left(x_{n} ; a_{n}\right)=I+\mathcal{O}\left(n^{-\frac{1}{2}}\right) .
$$

It also follows from (4.9) that

$$
n^{\frac{\beta}{2}} E_{n}\left(x_{n} ; a_{n}\right)=\mathcal{O}\left(n^{\frac{1}{2}}\right), \quad n^{-\frac{\beta}{2}} E_{n}\left(y_{n} ; a_{n}\right)^{-1}=\mathcal{O}\left(n^{\frac{1}{2}}\right) .
$$

A straightforward combination of (4.8), (4.11), and (4.12), would yield an $\mathcal{O}(1)$ bound for the left-hand side of (3.11). We need to go a little deeper into the structure of $E_{n}$ in order to improve the bound.

From the computations in Sec. VI of Ref. 13 it follows that $E_{n}\left(x_{n} ; a_{n}\right)$ has the following behavior as $n \rightarrow \infty$ :

$$
E_{n}\left(x_{n} ; a_{n}\right)=C_{1} n^{\frac{1-\beta}{2}} D_{\infty}^{-1}\left(\begin{array}{c}
\sqrt{2} i \\
1 \\
-1
\end{array}\right)\left(\begin{array}{lll}
0 & 0 & 1
\end{array}\right)\left(I+\mathcal{O}\left(n^{-\frac{1}{2}}\right)\right)
$$


where $C_{1}$ is some non-zero constant, see Eq. (6.12) of Ref. 13. From similar computations one finds for some non-zero $C_{2}$,

$$
E_{n}\left(y_{n} ; a_{n}\right)^{-1}=C_{2} n^{\frac{\beta+1}{2}}\left(\begin{array}{l}
1 \\
0 \\
0
\end{array}\right)\left(\begin{array}{lll}
\sqrt{2} i & 1 & -1
\end{array}\right) D_{\infty}\left(I+\mathcal{O}\left(n^{-\frac{1}{2}}\right)\right) .
$$

From these expansions it follows that the leading terms of $E_{n}\left(y_{n} ; a_{n}\right)$ and $E_{n}\left(x_{n} ; a_{n}\right)$ cancel out with $V_{n}$, see (4.4). In particular it holds by (4.4), (4.6), (4.13), and (4.14) that

$$
E_{n}\left(y_{n} ; a_{n}\right)^{-1}\left[V_{n}\left(y_{n} ; a_{n}\right)-V_{n}\left(x_{n} ; a_{n}\right)\right] E_{n}\left(x_{n}\right)=\mathcal{O}\left(n^{-1}\right) \text { as } n \rightarrow \infty .
$$

Taking into account the extra estimate (4.15) we can perform the following computation (we do not write $a_{n}$, and we use (4.1), (4.7), (4.5), (4.11), and the fact that $V_{n}\left(x_{n}\right)$ and $V_{n}\left(y_{n}\right)$ are uniformly bounded)

$$
\begin{aligned}
E_{n}\left(y_{n}\right)^{-1} R_{0}\left(y_{n}\right)^{-1} R_{0}\left(x_{n}\right) E_{n}\left(x_{n}\right) & \\
& =E_{n}\left(y_{n}\right)^{-1}\left(I+V_{n}\left(y_{n}\right)\right) R\left(y_{n}\right)^{-1} R\left(x_{n}\right)\left(I-V_{n}\left(x_{n}\right)\right) E_{n}\left(x_{n}\right) \\
& =E_{n}\left(y_{n}\right)^{-1}\left(I+V_{n}\left(y_{n}\right)\right)\left[I+\mathcal{O}\left(n^{-\frac{7}{6}}\right)\right]\left(I-V_{n}\left(x_{n}\right)\right) E_{n}\left(x_{n}\right) \\
& =E_{n}\left(y_{n}\right)^{-1}\left[I+V_{n}\left(y_{n}\right)-V_{n}\left(x_{n}\right)+\mathcal{O}\left(n^{-\frac{7}{6}}\right)\right] E_{n}\left(x_{n}\right) \\
& =I+\mathcal{O}\left(n^{-\frac{1}{6}}\right)
\end{aligned}
$$

which completes the proof of Proposition 3.2.

\section{PROOF OF PROPOSITIONS 1.2 AND 1.3}

In Sec. IV we derived expressions for $\mathbb{K}^{\text {Ang }}$ in terms of $\Psi$, see right-hand side of (3.12). In the present section these expressions will be used to obtain the explicit formulas stated in Propositions 1.2 and 1.3. We follow here the computations in Sec. 10.2 Ref. 6, see also Sec. 8.2 of Ref. 23.

As stated in Sec. II B the entries of $\Psi$ are solutions and derivatives of solutions to the third order differential equation

$$
z q^{\prime \prime \prime}(z)-\beta q^{\prime \prime}(z)-\tau q^{\prime}(z)+q(z)=0 .
$$

The solution functions $q_{j}(z), j=1,2,3$ have explicit expressions

$$
q_{j}(z)=\int_{\Gamma_{j}} t^{-\beta-3} e^{\frac{\tau}{t}-\frac{1}{2 t^{2}}+z t} d t
$$

with contours $\Gamma_{j}$ as shown in Figure 6.

A first step towards the derivation of the double integral formula consists of finding similar explicit expressions for the entries of the inverse of $\Psi$. This involves an adjoint differential equation to the one for the $q$-functions (5.1), and an associated bilinear concomitant, see, e.g., Refs. 3 and 18.

The solutions of this adjoint differential equation have contour integral representations, and it takes some work to identify the appropriate contours. When the explicit expressions for the entries of $\Psi^{-1}$ are known we will be in a position to prove the double integral formula for $\mathbb{K}^{\text {Ang }}$.

The adjoint differential equation reads

$$
z r^{\prime \prime \prime}(z)+(\beta+3) r^{\prime \prime}(z)-\tau r^{\prime}(z)-r(z)=0 .
$$


The associated bilinear concomitant $\mathcal{B}$ is a differential operator defined on general twice differentiable functions $q$ and $r$,

$$
\begin{array}{r}
\mathcal{B}[q, r](x, y):=y r(y) q^{\prime \prime}(x)-\left[(\beta+1) r(y)+y r^{\prime}(y)\right] q^{\prime}(x) \\
+\left[y r^{\prime \prime}(y)+(\beta+2) r^{\prime}(y)-\tau r(y)\right] q(x) .
\end{array}
$$

In order to ease the notation we introduce 3 operators $\mathcal{B}_{0}, \mathcal{B}_{1}$, and $\mathcal{B}_{2}$

$$
\begin{aligned}
& \mathcal{B}_{0} r(z):=z r^{\prime \prime}(z)+(\beta+2) r^{\prime}(z)-\tau r(z), \\
& \mathcal{B}_{1} r(z):=-z r^{\prime}(z)-(\beta+1) r(z), \\
& \mathcal{B}_{2} r(z):=z r(z) .
\end{aligned}
$$

So we can write the bilinear concomitant as

$$
\mathcal{B}[q, r](x, y)=\mathcal{B}_{2} r(y) q^{\prime \prime}(x)+\mathcal{B}_{1} r(y) q^{\prime}(x)+\mathcal{B}_{0} r(y) q(x) .
$$

The central property of this bilinear concomitant is that if $q$ solves the differential equation for $q$ (5.1) and $r$ solves the differential equation for $r$ (5.3), then the bilinear concomitant evaluated on the diagonal $\mathcal{B}[q, r](x, x)$ is a constant. This can be checked by taking the derivative of $\mathcal{B}[q, r](x, x)$ with respect to $x$ and plugging in the differential identities for $q$ and $r$.

The differential equation for $r$ (5.1) allows solutions in the form of a contour integral: for an appropriate contour $\widehat{\Gamma}$ the function

$$
r(z):=\int_{\widehat{\Gamma}} s^{\beta} e^{-\frac{\tau}{s}+\frac{1}{2 s^{2}}-z s} d s
$$

solves the differential equation (5.3). By an appropriate contour is meant a contour such that the integrand vanishes exponentially fast at the endpoints. In this case this implies that $\widehat{\Gamma}$ must end and start in 0 or at $\infty$.

Now we construct solutions $r_{k}(z), k=1,2,3$ to (5.3) such that

$$
\mathcal{B}\left[q_{j}, r_{k}\right](z, z)=\delta_{j k} \quad j, k=1,2,3,
$$

where we put the cuts of the functions $r_{k}$, if any, on the negative real axis. Using these relations we can find expressions for $\Psi^{-1}$. In each sector of the complex plane $\Psi(z)$ is of the form

$$
\Psi(z)=\left(\begin{array}{ccc}
q_{1}(z) & q_{2}(z) & q_{3}(z) \\
q_{1}^{\prime}(z) & q_{2}^{\prime}(z) & q_{3}^{\prime}(z) \\
q_{1}^{\prime \prime}(z) & q_{2}^{\prime \prime}(z) & q_{3}^{\prime \prime}(z)
\end{array}\right) \cdot C
$$

for some invertible matrix $C$. The inverse of $\Psi$ is then given by

$$
\Psi(z)^{-1}=C^{-1} \cdot\left(\begin{array}{lll}
\mathcal{B}_{0} r_{1}(z) & \mathcal{B}_{1} r_{1}(z) & \mathcal{B}_{2} r_{1}(z) \\
\mathcal{B}_{0} r_{2}(z) & \mathcal{B}_{1} r_{2}(z) & \mathcal{B}_{2} r_{2}(z) \\
\mathcal{B}_{0} r_{3}(z) & \mathcal{B}_{1} r_{3}(z) & \mathcal{B}_{2} r_{3}(z)
\end{array}\right)
$$

Indeed, the product $\Psi(z)^{-1} \Psi(z)$ then equals

$$
C^{-1} \cdot\left(\begin{array}{lll}
\mathcal{B}\left[q_{1}, r_{1}\right](z, z) & \mathcal{B}\left[q_{2}, r_{1}\right](z, z) & \mathcal{B}\left[q_{3}, r_{1}\right](z, z) \\
\mathcal{B}\left[q_{1}, r_{2}\right](z, z) & \mathcal{B}\left[q_{2}, r_{2}\right](z, z) & \mathcal{B}\left[q_{3}, r_{2}\right](z, z) \\
\mathcal{B}\left[q_{1}, r_{3}\right](z, z) & \mathcal{B}\left[q_{2}, r_{3}\right](z, z) & \mathcal{B}\left[q_{3}, r_{3}\right](z, z)
\end{array}\right) \cdot C,
$$

which by (5.8) is the identity matrix.

The functions $r_{k}(z)$ can be chosen to be of the form

$$
r_{k}(z)=\frac{1}{2 \pi i} \int_{\widehat{\Gamma}_{k}} s^{\beta} e^{-\frac{\tau}{s}+\frac{1}{2 s^{2}}-z s} d s,
$$

with the contours $\widehat{\Gamma}_{k}$ determined by the following proposition: 
Proposition 5.1: Let $\Omega \subset \mathbb{C}$ be a non-empty open set. Assume that the following two contour integrals converge for $z \in \Omega$ :

$$
q(z)=\int_{\Gamma} t^{-\beta-3} e^{\frac{\tau}{t}-\frac{1}{2 t^{2}}+z t} d t, \quad r(z)=\int_{\widehat{\Gamma}} s^{\beta} e^{-\frac{\tau}{s}+\frac{1}{2 s^{2}}-z s} d s .
$$

Also assume that the cuts of $t^{-\beta-3}$ and $s^{\beta}$ coincide, such that $t^{-\beta-3} s^{\beta}=t^{-3}$ for $t=s$.

If $\Gamma$ and $\widehat{\Gamma}$ do not intersect, or only intersect in 0 , then $\mathcal{B}[q, r](z, z)=0$ for $z \in \Omega$. If they however intersect once transversally at a non-zero point, and if $\Gamma$ meets $\widehat{\Gamma}$ on the negative side of $\widehat{\Gamma}$, then

$$
\mathcal{B}[q, r](z, z)=2 \pi i, \quad z \in \Omega .
$$

Proof: In order to simplify the notations we introduce the two phase functions $\theta_{q}$ and $\theta_{r}$,

$$
\begin{gathered}
\theta_{q}(t):=\frac{\tau}{t}-\frac{1}{2 t^{2}}+z t-\beta \log t, \\
\theta_{r}(s):=-\frac{\tau}{s}+\frac{1}{2 s^{2}}-z s+(\beta+3) \log s .
\end{gathered}
$$

Using the definition of the bilinear concomitant (5.4) and the definitions of $q(z)$ and $r(z)$ (5.13) we can write the concomitant $\mathcal{B}[q, r](z, z)$ of $q$ and $r$ as a double integral,

$$
\int_{t \in \Gamma} \int_{s \in \widehat{\Gamma}} \frac{z\left(s^{2}+s t+t^{2}\right)-(\beta+2) s-(\beta+1) t-\tau}{s^{3} t^{3}} e^{\theta_{q}(t)} e^{\theta_{r}(s)} d s d t .
$$

The fraction in the above integral can be split up as follows:

$$
\begin{aligned}
& \frac{z\left(s^{2}+s t+t^{2}\right)-(\beta+2) s-(\beta+1) t-\tau}{s^{3} t^{3}} \\
& \quad=\frac{1}{t^{3}(t-s)} \frac{\partial \theta_{r}(s)}{\partial s}+\frac{1}{s^{3}(t-s)} \frac{\partial \theta_{q}(t)}{\partial t}-\frac{s^{2}+s t+t^{2}}{s^{3} t^{3}(t-s)} .
\end{aligned}
$$

Suppose that the contours $\Gamma$ and $\widehat{\Gamma}$ do not intersect, or only intersect in 0 . Then the three terms on the right-hand side of (5.18) remain bounded and we can write

$$
\begin{aligned}
\mathcal{B}[q, r](z, z) & \\
= & \int_{t \in \Gamma} \int_{s \in \widehat{\Gamma}} \frac{1}{t^{3}(t-s)} \frac{\partial \theta_{r}(s)}{\partial s} e^{\theta_{q}(t)} e^{\theta_{r}(s)} d s d t \\
& +\int_{t \in \Gamma} \int_{s \in \widehat{\Gamma}} \frac{1}{s^{3}(t-s)} \frac{\partial \theta_{q}(t)}{\partial t} e^{\theta_{q}(t)} e^{\theta_{r}(s)} d s d t \\
& -\int_{t \in \Gamma} \int_{s \in \widehat{\Gamma}} \frac{s^{2}+s t+t^{2}}{s^{3} t^{3}(t-s)} e^{\theta_{q}(t)} e^{\theta_{r}(s)} d s d t .
\end{aligned}
$$




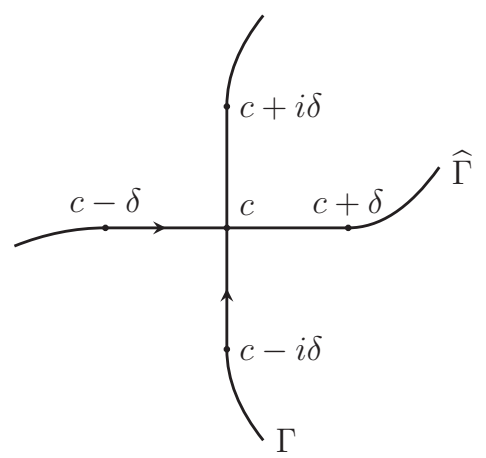

FIG. 7. The deformed contours $\Gamma$ containing the interval $[c-i \delta, c+i \delta]$ and $\widehat{\Gamma}$ containing $[c-\delta, c+\delta]$.

We can apply integration by parts to the first two integrals in (5.19). The boundary terms vanish. What remains is

$$
\begin{aligned}
\mathcal{B}[q, r](z, z)= & -\int_{t \in \Gamma} \int_{s \in \widehat{\Gamma}} \frac{1}{t^{3}(t-s)^{2}} e^{\theta_{q}(t)} e^{\theta_{r}(s)} d s d t \\
& +\int_{t \in \Gamma} \int_{s \in \widehat{\Gamma}} \frac{1}{s^{3}(t-s)^{2}} e^{\theta_{q}(t)} e^{\theta_{r}(s)} d s d t \\
& -\int_{t \in \Gamma} \int_{s \in \widehat{\Gamma}} \frac{t^{3}-s^{3}}{s^{3} t^{3}(t-s)^{2}} e^{\theta_{q}(t)} e^{\theta_{r}(s)} d s d t \\
= & 0 .
\end{aligned}
$$

Now suppose that $\Gamma$ and $\widehat{\Gamma}$ intersect in a point $c \neq 0$, with $\Gamma$ going from the right side of $\widehat{\Gamma}$ to the left side. Then the contours can be deformed such that $\Gamma$ contains the interval $[c-i \delta$, $c+i \delta]$ while $\widehat{\Gamma}$ contains $[c-\delta, c+\delta]$ for some $\delta>0$, see Figure 7. Define a new contour $\widehat{\Gamma}^{\varepsilon}$ by $\widehat{\Gamma}^{\varepsilon}:=\widehat{\Gamma} \backslash[c-\varepsilon, c+\varepsilon]$ with $0<\varepsilon<\delta$. Then we have, using (5.17),

$$
\mathcal{B}[q, r](z, z)=\lim _{\varepsilon \rightarrow 0^{+}} \int_{t \in \Gamma} \int_{s \in \widehat{\Gamma}^{\varepsilon}}\left[\begin{array}{c}
z\left(s^{2}+s t+t^{2}\right)-\tau \\
+(\beta+2) s-(\beta+1) t
\end{array}\right] \frac{e^{\theta_{q}(t)} e^{\theta_{r}(s)}}{s^{3} t^{3}} d s d t .
$$

Since $\widehat{\Gamma}^{\varepsilon}$ and $\Gamma$ do not intersect we can use the expression in (5.19) with $\widehat{\Gamma}$ replaced by $\widehat{\Gamma}^{\varepsilon}$ and apply integration by parts. However, now the boundary term in $s$ remains,

$$
\mathcal{B}[q, r](z, z)=\lim _{\varepsilon \rightarrow 0^{+}} \int_{\Gamma} \frac{1}{t^{3}}\left[\frac{1}{t-s} e^{\theta_{r}(s)}\right]_{s=c+\varepsilon}^{s=c-\varepsilon} e^{\theta_{q}(t)} d t .
$$

By continuity we have $e^{\theta_{r}(c \pm \varepsilon)}=e^{\theta_{r}(c)}+\mathcal{O}(\varepsilon)$ as $\varepsilon \rightarrow 0$. Hence we obtain

$$
\mathcal{B}[q, r](z, z)=\lim _{\varepsilon \rightarrow 0}\left(e^{\theta_{r}(c)}+\mathcal{O}(\varepsilon)\right) \int_{\Gamma} \frac{1}{t^{3}}\left[\frac{1}{t-c+\varepsilon}-\frac{1}{t-c-\varepsilon}\right] e^{\theta_{q}(t)} d t .
$$

Finally, we deform the segment $[c-i \delta, c+i \delta] \subset \Gamma$ into the union of a small circle around $c$ $+\varepsilon$ and a semicircle with radius $\delta$ around $c$, see Figure 8. The small circle around $c+\varepsilon$ will give us a residue contribution, while the contribution of the semicircle and the remaining part of $\Gamma$ tends to zero as $\varepsilon \rightarrow 0^{+}$. Remark that the small circle around $c+\varepsilon$ has clockwise orientation. Then we have as $\varepsilon \rightarrow 0^{+}$

$$
\int_{\Gamma} \frac{1}{t^{3}}\left[\frac{1}{t-c+\varepsilon}-\frac{1}{t-c-\varepsilon}\right] e^{\theta_{q}(t)} d t=2 \pi i \frac{1}{(c+\varepsilon)^{3}} e^{\theta_{q}(c+\varepsilon)}+\mathcal{O}(\varepsilon) .
$$




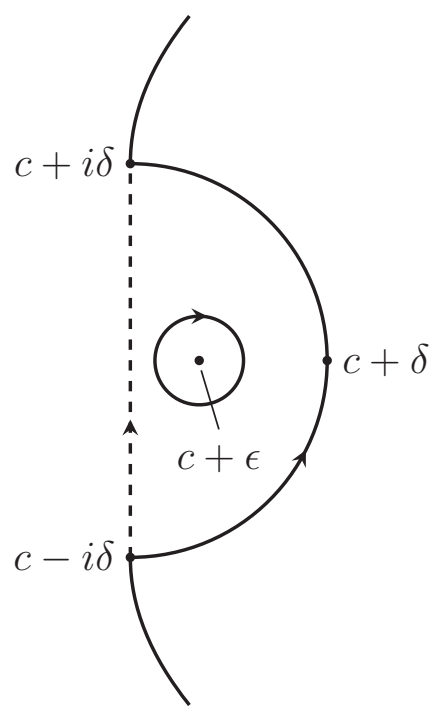

FIG. 8. The dashed part of the contour $\Gamma$ is deformed into a semicircle of radius $\delta$ around $c$. Additionally, a residue is picked up in $c+\varepsilon$. The contour $\widehat{\Gamma}$ is not shown here.

Taking the limit $\varepsilon \rightarrow 0^{+}$, and plugging the result into (5.23) we get

$$
\mathcal{B}[q, r](z, z)=2 \pi i \frac{1}{c^{3}} e^{\theta_{q}(c)} e^{\theta_{r}(c)}=2 \pi i,
$$

where we used (5.15) and (5.16).

Choose contours $\widehat{\Gamma}_{k}$ as in Figure 9 and let the functions $r_{k}$ be defined by

$$
r_{k}(z):=\frac{1}{2 \pi i} \int_{\widehat{\Gamma}_{k}} s^{\beta} e^{-\frac{\tau}{s}+\frac{1}{2 s^{2}}-z s} d s, \quad k=1,2,3,
$$

where $s^{\beta}$ has a cut on the positive real axis and $s_{+}^{\beta}=|s|^{\beta}$. Then it follows from Proposition 5.1 that the concomitant conditions (5.8) are satisfied. The integrals (5.26) define analytic functions on $\mathbb{C}$ with possible branch points in 0 and $\infty$ and we put the branch cut on the negative real axis.

Proof of Proposition 1.2: Having found explicit expressions for the entries of $\Psi^{-1}$ we can return to the expression for $\mathbb{K}^{\text {Ang }}$ given in (3.12). The $1 \times 3$ and $3 \times 1$ matrices pick out certain linear combinations of the $q_{j}$ and $r_{k}$, and we obtain

$$
\mathbb{K}^{\mathrm{Ang}}(x, y ; \tau)=\frac{1}{(x-y)}\left|\frac{y}{x}\right|^{\beta} \mathcal{B}\left[q_{0}, r_{0}\right](x, y),
$$

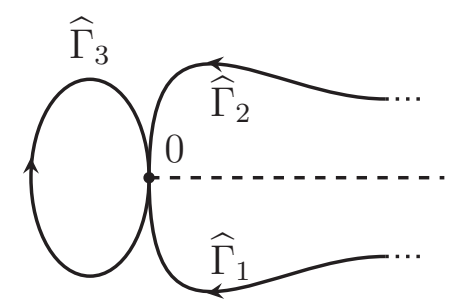

FIG. 9. The contours $\widehat{\Gamma}_{k}, k=1,2,3$. The dashed line denotes the cut of $s^{\beta}$. 
with $q_{0}$ and $r_{0}$ given by

$$
\begin{gathered}
q_{0}(x):=\frac{1}{2 \pi i} \cdot\left\{\begin{array}{cc}
e^{2 \beta \pi i} q_{1}(x)+q_{2}(x) & \text { for } x>0, \\
e^{\beta \pi i} q_{1,+}(x)+e^{-\beta \pi i} q_{2,+}(x) & \text { for } x<0,
\end{array}\right. \\
r_{0}(y):=\left\{\begin{array}{cl}
r_{2}(y)-e^{-2 \beta \pi i} r_{1}(y) & \text { for } y>0, \\
e^{-\beta \pi i} r_{3}(y) & \text { for } y<0 .
\end{array}\right.
\end{gathered}
$$

The functions $q_{1}$ and $q_{2}$ have branch cuts on the negative real axis. Hence we have to specify that we need the positive boundary value in (5.28) in the case $x<0$. Using contour deformation and a simple substitution in the integral one can see that $q_{0}$ and $r_{0}$ are given by the contour integrals (1.15) and (1.16).

Finally we derive from (5.27) the double integral formula.

Proof of Proposition 1.3: One can check that the derivatives of $q_{0}$ and $r_{0}$ have the following expressions:

$$
\begin{gathered}
q_{0}^{(n)}(x)=\frac{1}{2 \pi i} x^{2-n}|x|^{\beta} \int_{\Gamma_{0}} t^{-\beta+n-3} e^{\frac{\tau x}{t}-\frac{x^{2}}{2 t^{2}}+t} d t, \\
r_{0}^{(n)}(y)=\frac{(-1)^{n}}{2 \pi i} y^{2-n}|y|^{-\beta-3} \int_{\widehat{\Gamma}_{0}} s^{\beta+n} e^{-\frac{\tau y}{s}+\frac{y^{2}}{2 s^{2}}-s} d s,
\end{gathered}
$$

for $n \in \mathbb{Z}^{+}$and $x, y \in \mathbb{R} \backslash\{0\}$. The contours $\Gamma_{0}$ and $\widehat{\Gamma}_{0}$ are given in Figure 3. From (5.27) and the definition of the bilinear concomitant (5.4) it follows that we can write

$$
\begin{aligned}
& \mathbb{K}^{\mathrm{Ang}}(x, y ; \tau)=\frac{1}{4 \pi^{2}(y-x)|y|^{3}} \\
& \cdot \int_{t \in \Gamma_{0}} \int_{s \in \widehat{\Gamma}_{0}} \frac{s^{\beta}}{t^{\beta+3}}\left[\begin{array}{c}
y^{3} t^{2}-(\beta+1) x y^{2} t+x y^{2} s t \\
+x^{2} y s^{2}-(\beta+2) x^{2} y s-\tau x^{2} y^{2}
\end{array}\right] e^{\frac{e^{\frac{\tau x}{t}}-\frac{x^{2}}{2 t^{2}}+t}{s}-\frac{y^{2}}{2 s^{2}}+s} d s d t .
\end{aligned}
$$

Introducing the phase functions $\eta_{q}$ and $\eta_{r}$

$$
\begin{gathered}
\eta_{q}(t):=-\beta \log t+\frac{\tau x}{t}-\frac{x^{2}}{2 t^{2}}+t, \\
\eta_{r}(s):=(\beta+3) \log s-\frac{\tau y}{s}+\frac{y^{2}}{2 s^{2}}-s,
\end{gathered}
$$

we can rewrite the double integral in (5.32) as

$$
\int_{t \in \Gamma_{0}} \int_{s \in \widehat{\Gamma}_{0}} \frac{1}{s^{3} t^{3}}\left[\begin{array}{c}
y^{3} t^{2}-(\beta+1) x y^{2} t+x y^{2} s t \\
+x^{2} y s^{2}-(\beta+2) x^{2} y s-\tau x^{2} y^{2}
\end{array}\right] e^{\eta_{q}(t)} e^{\eta_{r}(s)} d s d t .
$$

We have the following straightforward identity for the expression inside the square brackets:

$$
\begin{aligned}
y^{3} t^{2}- & (\beta+1) x y^{2} t+x y^{2} s t+x^{2} y s^{2}-(\beta+2) x^{2} y s-\tau x^{2} y^{2} \\
& =-\frac{x^{3} y s^{3}}{x s-y t} \frac{\partial \eta_{r}(s)}{\partial s}-\frac{x y^{3} t^{3}}{x s-y t} \frac{\partial \eta_{q}(t)}{\partial t} \\
& +\frac{x^{4} y s^{3}-x y^{4} t^{3}}{(x s-y t)^{2}}+y^{3} t^{3} \frac{x-y}{x s-y t} .
\end{aligned}
$$


The terms involving derivatives of the phase functions $\eta_{q}$ and $\eta_{r}$ can be simplified using integration by parts,

$$
\begin{aligned}
& \int_{t \in \Gamma_{0}} \int_{s \in \widehat{\Gamma}_{0}} \frac{1}{s^{3} t^{3}} \frac{x^{3} y s^{3}}{x s-y t} \frac{\partial \eta_{r}(s)}{\partial s} e^{\eta_{q}(t)} e^{\eta_{r}(s)} d s d t \\
& =\int_{t \in \Gamma_{0}} \int_{s \in \widehat{\Gamma}_{0}} \frac{1}{s^{3} t^{3}} \frac{x^{4} y s^{3}}{(x s-y t)^{2}} e^{\eta_{q}(t)} e^{\eta_{r}(s)} d s d t \\
& \int_{t \in \Gamma_{0}} \int_{s \in \widehat{\Gamma}_{0}} \frac{1}{s^{3} t^{3}} \frac{x y^{3} t^{3}}{x s-y t} \frac{\partial \eta_{q}(t)}{\partial t} e^{\eta_{q}(t)} e^{\eta_{r}(s)} d s d t \\
& =-\int_{\Gamma_{0}} \int_{\widehat{\Gamma}_{0}} \frac{1}{s^{3} t^{3}} \frac{x y^{4} t^{3}}{(x s-y t)^{2}} e^{\eta_{q}(t)} e^{\eta_{r}(s)} d s d t
\end{aligned}
$$

By plugging (5.37) and (5.38) together with (5.36) into (5.35) we find

$$
\begin{gathered}
\int_{t \in \Gamma_{0}} \int_{s \in \widehat{\Gamma}_{0}} \frac{1}{s^{3} t^{3}}\left[\begin{array}{c}
y^{3} t^{2}-(\beta+1) x y^{2} t+x y^{2} s t \\
+x^{2} y s^{2}-(\beta+2) x^{2} y s-\tau x^{2} y^{2}
\end{array}\right] e^{\eta_{q}(t)} e^{\eta_{r}(s)} d s d t \\
=(x-y) \int_{t \in \Gamma_{0}} \int_{s \in \widehat{\Gamma}_{0}} \frac{y^{3}}{s^{3}(x s-y t)} e^{\eta_{q}(t)} e^{\eta_{r}(s)} d s d t .
\end{gathered}
$$

From (5.32) we then conclude that $\mathbb{K}^{\operatorname{Ang}}(x, y ; \tau)$ is given by

$$
\mathbb{K}^{\mathrm{Ang}}(x, y ; \tau)=\frac{\operatorname{sgn}(y)}{(2 \pi i)^{2}} \int_{t \in \Gamma_{0}} \int_{s \in \widehat{\Gamma}_{0}} \frac{s^{\beta}}{t^{\beta}} \frac{1}{x s-y t} \frac{e^{\frac{\tau x}{t}-\frac{x^{2}}{2 t^{2}}+t}}{e^{\frac{\tau y}{s}-\frac{y^{2}}{2 s^{2}}+s}} d s d t .
$$

\section{ACKNOWLEDGMENTS}

The authors are supported by K.U. Leuven Research Grant No. OT/0833, and by the Belgian Interuniversity Attraction Pole P06/02.

The second author is also supported by FWO-Flanders projects G.0427.09 and G.0641.11, and by Grant No. MTM2008-06689-C02-01 of the Spanish Ministry of Science and Innovation.

${ }^{1}$ A. Angelesco, "Sur deux extensions des fractions continues algébriques," C. R. Acad. Sci. Paris 168, 262-265 (1919).

${ }^{2}$ A. I. Aptekarev, P. M. Bleher, and A. B. J. Kuijlaars, "Large $n$ limit of Gaussian random matrices with external source: part II," Commun. Math. Phys. 256, 367-389 (2005).

${ }^{3}$ M. Bertola, "Biorthogonal polynomials for two-matrix models with semiclassical potentials," J. Approx. Theory 144, 162-212 (2007).

${ }^{4}$ P. M. Bleher and A. B. J. Kuijlaars, "Random matrices with external source and multiple orthogonal polynomials," Int. Math. Res. Notices 3, 109-129 (2004).

${ }^{5}$ P. M. Bleher and A. B. J. Kuijlaars, "Large $n$ limit of Gaussian random matrices with external source: part I," Commun. Math. Phys. 252, 43-76 (2004).

${ }^{6}$ P. M. Bleher and A. B. J. Kuijlaars, "Large $n$ limit of Gaussian random matrices with external source, part III: double scaling limit," Commun. Math. Phys. 270, 481-517 (2007).

${ }^{7}$ A. Borodin, "Biorthogonal ensembles," Nucl. Phys. B 536, 704-732 (1999).

${ }^{8}$ E. Brézin and S. Hikami, "Universal singularity at the closure of a gap in a random matrix theory," Phys. Rev. E 57, 4140-4149 (1998).

${ }^{9}$ T. Claeys and A. Kuijlaars, "Universality in unitary random matrix ensembles when the soft edge meets the hard edge," Integrable Systems and Random Matrices: In Honor of Percy Deift, Contemporary Mathematics Vol. 458 (American Mathematical Society, Providence, RI, 2008), pp. 265-280.

${ }^{10}$ E. Daems and A. B. J. Kuijlaars, "A Christoffel-Darboux formula for multiple orthogonal polynomials," J. Approx. Theory 130, 188-200 (2004).

${ }^{11}$ P. Deift, Orthogonal Polynomials and Random Matrices: A Riemann-Hilbert Approach, Courant Lecture Notes in Mathematics Vol. 3 (American Mathematical Society, Providence, RI, 1999). 
${ }^{12}$ P. Deift, T. Kriecherbauer, K. T.-R. McLaughlin, S. Venakides, and X. Zhou, "Uniform asymptotics for polynomials orthogonal with respect to varying exponential weights and applications to universality questions in random matrix theory," Commun. Pure Appl. Math. 52, 1335-1425 (1999).

${ }^{13}$ K. Deschout and A. B. J. Kuijlaars, "Double scaling limit for modified Jacobi-Angelesco polynomials," in Notions of Positivity and the Geometry of Polynomials, Trends in Mathematics, edited by P. Brändén, M. Passare, and M. Putinar (Springer, Basel, 2011), pp. 115-161.

${ }^{14}$ M. Duits and D. Geudens, “A critical phenomenon in the two-matrix model in the quartic/quadratic case," Duke Math. J. (to be published); preprint arXiv:1111.2162.

${ }^{15}$ P. J. Forrester, Log-gases and Random Matrices (Princeton University Press, Princeton, NJ, 2010).

${ }^{16}$ M. Gaudin, "Sur la loi limite de l'espacement des valeurs propres d'une matrice aléatoire," Nucl. Phys. 25, 447-458 (1961).

${ }^{17}$ M. Gaudin and M. L. Mehta, "On the density of eigenvalues of a random matrix," Nucl. Phys. 18, 420-427 (1960).

${ }^{18}$ E. L. Ince, Ordinary Differential Equations (Dover, New York, 1944).

${ }^{19}$ V. A. Kaliaguine, "On a class of polynomials defined by two orthogonality conditions," Math. USSR Sb. 38, 563-580 (1981) [Mat. Sb. 110, 609-627 (1979) (in Russian)].

${ }^{20}$ W. König, "Orthogonal polynomial ensembles in probability theory," Probab. Surv. 2, 385-447 (2005).

${ }^{21}$ A. B. J. Kuijlaars, "Multiple orthogonal polynomial ensembles," in Recent Trends in Orthogonal Polynomials and Approximation Theory, Contemporary Mathematics Vol. 507, edited by J. Arvesú et al. (American Mathematical Society, 2010), pp. $155-176$.

${ }^{22}$ A. B. J. Kuijlaars, A. Martínez-Finkelshtein, and F. Wielonsky, "Non-intersecting squared Bessel paths and multiple orthogonal polynomials for modified Bessel weights," Commun. Math. Phys. 286, 217-275 (2009).

${ }^{23}$ A. B. J. Kuijlaars, A. Martínez-Finkelshtein, and F. Wielonsky, "Non-intersecting squared Bessel paths: critical time and double scaling limit," Commun. Math. Phys. 308, 227-279 (2011).

${ }^{24}$ A. B. J. Kuijlaars, K. T.-R. McLaughlin, W. Van Assche, and M. Vanlessen, "The Riemann-Hilbert approach to strong asymptotics for orthogonal polynomials on [-1, 1]," Adv. Math. 188, 337-398 (2004).

${ }^{25}$ M. L. Mehta, "On the statistical properties of the level-spacings in nuclear spectra," Nucl. Phys. 18, 395-419 (1960).

${ }^{26}$ E. M. Nikishin and V. N. Sorokin, Rational Approximations and Orthogonality, Translations of Mathematical Monographs Vol. 92 (American Mathematical Society, Providence, RI, 1991).

${ }^{27}$ T. Takata, "Certain multiple orthogonal polynomials and a discretization of the Bessel equation," J. Math. Kyoto Univ. 49, 747-769 (2009).

${ }^{28}$ D. N. Tulyakov, "Difference equations having bases with power-like growth which are perturbed by a spectral parameter," Sb. Math. 200, 753-781 (2009).

${ }^{29}$ C. Tracy and H. Widom, "The Pearcey process," Commun. Math. Phys. 263, 381-400 (2006).

${ }^{30}$ W. Van Assche, J. S. Geronimo, and A. B. J. Kuijlaars, "Riemann-Hilbert problems for multiple orthogonal polynomials," in Special Functions 2000: Current Perspective and Future Directions, edited by J. Bustoz et al. (Kluwer, Dordrecht, 2001), pp. 23-59. 